\title{
Calcul fonctionnel harmonique dans une algebre de Banach involutive quotient
}

\author{
M. Akkar \\ H. Arroub
}

\begin{abstract}
We use the Poisson integral formula in order to establish harmonic functional calculus for quotient involutive Banach algebras. We give several properties of this functional calculus. L.Waelbroeck's holomorphic functional calculus is a special case of ours. We use our harmonic functional calculus to extend result of J.W.M.Ford to quotient involutive Banach algebras.
\end{abstract}

L.WAELBROECK a montré dans [6] que le calcul fonctionnel holomorphe dans une algèbre de Banach $A$ se généralisait à une algèbre de Banach quotient $A / \alpha(\alpha$ un idéal de Banach de $A$ tel que l'injection $\alpha \longrightarrow A$ soit continue) et que c'est dans ce cadre qu'il est naturel et a de nombreuses applications .

Dans [2], l'auteur construit un calcul fonctionnel harmonique sur une algèbre de Banach involutive et donne quelques applications.

Nous étendons ici son calcul au cas quotient et montrons que ces applications (théorème de K.FAN et VON.NEUMANN) sont encore vraies dans le cas d'une algèbre de Banach quotient (voir [1])

\section{Introduction}

Soit $A$ une algèbre de Banach unitaire (non nécessairement commutative) involutive. Soit $\alpha$ un idéal bilatère auto-adjoint et distinct de $A$ tel que $\alpha$ est un sous-espace de Banach de $A$ (cf L. WAELBROECK [5]). $\alpha$ devient un idéal de Banach de $A$ (cf

Received by the editors November 1995 .

Communicated by J. Delanghe.

1991 Mathematics Subject Classification : Primary 46H, Secondary 46K.

Key words and phrases : quotient involutive Banach algebra, harmonic functional calculus. 
L.WAELBROECK [6]) et l'involution de $\alpha$ dans $\alpha$ devient continue. $A / \alpha$ est dite une algèbre de Banach involutive quotient.

Soit $\tilde{a}$ un élément de $A / \alpha$, le spectre de $\tilde{a}$ dans l'algèbre $A / \alpha$ est noté par $s p_{\alpha} a$. $s p_{\alpha} a$ est un compact non vide de $\mathbb{C}$.

On se donne un ouvert $U$ de $\mathbb{C}$ tel que $s p_{\alpha} a \subset B\left(z_{o}, R\right) \subset \bar{B}\left(z_{o}, R\right) \subset U$, $\left(z_{o} \in U, R>0\right)$. On désigne par $h(U, A)$ l'espace vectoriel complexe des applications $f: U \longrightarrow A$ harmoniques. $h(U, \alpha)$ est un sous-espace vectoriel de $h(U, A)$.

On pose $h(U, A / \alpha)=h(U, A) / h(U, \alpha)$. Un élément de $h(U, A / \alpha)$ est noté par $\tilde{f}$.

Le calcul fonctionnel harmonique dans l'algèbre de Banach involutive quotient $A / \alpha$ consiste à donner un sens à l'élément $\tilde{f}(\tilde{a})$ de $A / \alpha$ pour toute application $f: U \longrightarrow A$ harmonique. La construction de ce calcul fonctionnel harmonique est basée sur la formule intégrale de Poisson. L'application qui à $\tilde{f}$ associe $\tilde{f}(\tilde{a})$ est linéaire et vérifie :

1_ $\tilde{f}(\tilde{a})=\tilde{1}$ si $f=1$

2_ $\tilde{f}(\tilde{a})=\tilde{a}$ si $f=\mathbf{z}$ où $\mathbf{z}$ désigne l'application qui à $z$ associe $z$

3_ $\tilde{f}^{*}(\tilde{a})=(\tilde{f}(\tilde{a}))^{*}$ si $\tilde{a}$ commute avec $\tilde{f}$ et $\tilde{f}^{*}$.

Si $f$ est holomorphe on retrouve le calcul fonctionnel holomorphe défini par L.WAELBROECK [6].

Si $\alpha=0$ on retrouve le calcul fonctionnel harmonique défini par A.ELKINANI $[2]$.

\section{Préliminaires}

Dans toute la suite, un espace vectoriel ou une algèbre sera sur $\mathbb{C}$ et l'unité d'une algèbre sera notée par 1.

Nous rappelons tout d'abord quelques notions sur les structures quotients définies par L.WAELBROECK [4], [5], [6].

\subsection{Espace de Banach quotient [4], [5]}

Soient $(E,\|\|)$ un espace de Banach et $F$ un sous-espace vectoriel de $E$. F est dit un sous espace de Banach de $E$ si $F$ est muni d'une norme d'espace de Banach $n_{F}$ plus fine que celle induite par \|\| . Le théorème du graphe fermé permet de conclure que de telles normes $n_{F}$ sont équivalentes. On dit qu'il n'y a qu'une seule telle norme à une équivalence près. Le quotient $E / F$ est appelé espace de Banach quotient.

\subsection{Algèbre de Banach quotient $[6]$}

Soient $(A,\|\|)$ une algèbre de Banach unitaire et $\alpha$ un idéal bilatère distinct de $A$ tel que $\alpha$ soit un sous-espace de Banach de $A$. Soit $\mathrm{n}_{\alpha}$ une norme d'espace de Banach sur $\alpha$ plus fine que celle induite par \|\| .

Le théorème du graphe fermé permet de conclure que les multiplications 


$$
\begin{aligned}
& A \mathrm{x} \alpha \longrightarrow \alpha \quad \alpha \mathrm{x} A \longrightarrow \alpha \\
& (a, x) \longmapsto a x \quad(x, a) \longmapsto x a
\end{aligned}
$$

sont séparément continues, donc globalement continues.

On pose pour tout $x \in \alpha\|x\|_{\alpha}=\sup \left\{\mathrm{n}_{\alpha}(a x) \mid a \in A\|a\| \leq 1\right\}$.

\|\|$_{\alpha}$ est une norme d'espace de Banach sur $\alpha$ équivalente à $n_{\alpha}$.

D'autre part on a $\|a x\|_{\alpha} \leq\|a\|\|x\|_{\alpha}$ pour tout $a \in A$ et pour tout $x \in \alpha$, donc $\left(\alpha,\|\|_{\alpha}\right)$ est une algèbre de Banach.

$A / \alpha$ est dite une algèbre de Banach quotient.

Si de plus $A$ est à involution continue * et $\alpha$ est un idéal bilatère auto-adjoint de $A$ alors l'involution de $\alpha$ dans $\alpha$ est continue. $A / \alpha$ est dite une algèbre de Banach involutive quotient.

\subsection{Spectre dans une algèbre de Banach quotient}

Soient $A / \alpha$ une algèbre de Banach quotient et $\tilde{a}$ un élément de $A / \alpha$, le spectre de $\tilde{a}$ dans l'algèbre $A / \alpha$ sera noté par $s p_{\alpha} a$. Le spectre de $A$ dans $A$ sera noté par spa.

Proposition 1 : (voir aussi le cas où l'algèbre $A$ est commutative de L.WAELBROECK [6]).

Soient $A / \alpha$ une algèbre de Banach quotient et ã un élément de $A / \alpha$. Alors $s p_{\alpha} a$ est une partie non vide et compacte de $\mathbb{C}$.

\section{Preuve :}

$\alpha$ est contenu dans un idéal bilatère maximal $\alpha^{\prime}$ de $A$. Un tel idéal $\alpha^{\prime}$ est fermé dans $A . A / \alpha^{\prime}$ est une algèbre de Banach unitaire, donc $s p_{\alpha^{\prime}} a$ est non vide et est contenu dans $s p_{\alpha} a$ d'où $s p_{\alpha} a$ est non vide. D'autre part $s p_{\alpha} a$ est contenu dans $s p a$ qui est compact, donc $s p_{\alpha} a$ est borné. Pour conclure il suffit de prouver que $s p_{\alpha} a$ est fermé dans $\mathbb{C}$. En effet, soit $\lambda \in \mathbb{C} \backslash s p_{\alpha} a$ il existe $b_{\lambda} \in A, x_{\lambda} \in \alpha, y_{\lambda} \in \alpha$ tels que :

$$
(a-\lambda) b_{\lambda}+x_{\lambda}=b_{\lambda}(a-\lambda)+y_{\lambda}=1 .
$$

Le groupe des éléments inversibles dans $A$ est ouvert dans $A$. Donc il existe $V_{\lambda}$ un voisinage de $\lambda$ dans $\mathbb{C}$ tel que pour tout $z \in V_{\lambda}(a-z) b_{\lambda}+x_{\lambda}$ et $b_{\lambda}(a-z)+y_{\lambda}$ sont inversibles dans $A$.

Pour tout $z \in V_{\lambda}$ on pose :

$$
\begin{aligned}
& b_{z}=b \lambda\left(1-(z-\lambda) b_{\lambda}\right)^{-1} \in A \\
& x_{z}=x_{\lambda}\left(1-(z-\lambda) b_{\lambda}\right)^{-1} \in \alpha \\
& y_{z}=\left(1-(z-\lambda) b_{\lambda}\right)^{-1} y_{\lambda} \in \alpha
\end{aligned}
$$

On a $(a-z) b_{z}+x_{z}=b_{z}(a-z)+y_{z}=1$ pour tout $z \in V_{\lambda}$. D'où $V_{\lambda}$ est contenu dans $\mathbb{C} \backslash s p_{\alpha} a$.

Rappelons quelques notations.

Soient $A / \alpha$ une algèbre de Banach quotient et $U$ un ouvert de $\mathbb{R}^{n}(n \geq 1)$. On désigne par $\mathcal{C}^{p}(U, A),(p \geq 0)$ l'algèbre unitaire des applications $u: U \longrightarrow A$ de classe $\mathcal{C}^{p} \cdot \mathcal{C}^{p}(U, \alpha)$ est un idéal bilatère distinct de $\mathcal{C}^{p}(U, A)$. 
On pose : $\mathcal{C}^{p}(U, A / \alpha)=\mathcal{C}^{p}(U, A) / \mathcal{C}^{p}(U, \alpha)$. Un élément de l'algèbre $\mathcal{C}^{p}(U, A / \alpha)$ est noté par $\tilde{u}$ (la classe d'équivalence de $u$ modulo $\mathcal{C}^{p}(U, \alpha)$ ). Si de plus $A$ est involutive et $\alpha$ est un idéal bilatère auto-adjoint de $A$ alors $\mathcal{C}^{p}(U, \alpha)$ est un idéal bilatère auto-adjoint de l'algèbre involutive $\mathcal{C}^{p}(U, A)$. L'involution de $\mathcal{C}^{p}(U, A)$ dans lui même qui à $u$ associe $u^{*}$ envoie $\mathcal{C}^{p}(U, \alpha)$ dans lui même, donc elle induit une involution de l'algèbre $\mathcal{C}^{p}(U, A / \alpha)$ qui à $\tilde{u}$ associe $\tilde{u}^{*}$. Donc $\mathcal{C}^{p}(U, A / \alpha)$ devient une algèbre involutive unitaire.

On définit de même l'algèbre $\mathcal{C}^{\infty}(U, A / \alpha)=\mathcal{C}^{\infty}(U, A) / \mathcal{C}^{\infty}(U, \alpha)$ et l'involution $\tilde{u} \longmapsto \tilde{u}^{*}$ dans le cas où $A$ est involutive et $\alpha$ est un idéal bilatère auto-adjoint de $A$.

\section{Proposition 2 :}

Soient $A / \alpha$ une algèbre de Banach quotient et $\tilde{a}$ un élément de $A / \alpha$. Alors il existe $u: \mathbb{C} \backslash s p_{\alpha} a \longrightarrow A$ de classe $\mathcal{C}^{\infty}$ tel que $(\tilde{a}-\tilde{\mathbf{z}}) \tilde{u}=\tilde{u}(\tilde{a}-\tilde{\mathbf{z}})=\tilde{1}$ dans l'algèbre $\mathcal{C}^{\infty}\left(\mathbb{C} \backslash s p_{\alpha} a, A / \alpha\right)$ où $\mathbf{z}$ désigne l'application qui à $z$ associe $z$. Autrement dit $\tilde{a}-\tilde{\mathbf{z}}$ est inversible dans l'algèbre $\mathcal{C}^{\infty}\left(\mathbb{C} \backslash s p_{\alpha} a, A / \alpha\right)$.

\section{Preuve:}

On reprend la preuve de la proposition 1. Pour tout $\lambda \in \mathbb{C} \backslash s p_{\alpha} a$ il existe $V_{\lambda}$ un voisinage de ouvert de $\lambda$ dans $\mathbb{C}$ (on peut prendre une boule ouverte) tel que :

$$
\begin{gathered}
(a-z) b_{z}+x_{z}=b_{z}(a-z)+y_{z}=1 \text { pour tout } z \in V_{\lambda} \text { avec } \\
b_{z}=b_{\lambda}\left(1-(z-\lambda) b_{\lambda}\right)^{-1} \in A \\
x_{z}=x_{\lambda}\left(1-(z-\lambda) b_{\lambda}\right)^{-1} \in \alpha \\
y_{z}=\left(1-(z-\lambda) b_{\lambda}\right)^{-1} y_{\lambda} \in \alpha
\end{gathered}
$$

Pour tout $z \in V_{\lambda}$ on pose :

$$
u_{\lambda}(z)=b_{z}, v_{\lambda}(z)=x_{z}, w_{\lambda}(z)=y_{z}
$$

$u_{\lambda}: V_{\lambda} \longrightarrow A$ et $v_{\lambda}, w_{\lambda}: V_{\lambda} \longrightarrow \alpha$ sont analytiques donc de classe $\mathcal{C}^{\infty}$ et vérifient : $(a-z) u_{\lambda}(z)+v_{\lambda}(z)=u_{\lambda}(z)(a-z)+w_{\lambda}(z)=1$ pour tout $z \in V_{\lambda}$.

On pose $U=\mathbb{C} \backslash s p_{\alpha} a$ ouvert de $\mathbb{C}$. Les $V_{\lambda}$ forment un recouvrement d'ouverts de $U$. Le théorème d'existence d'une partition de l'unité de classe $\mathcal{C}^{\infty}$ subordonnée au recouvrement d'ouverts $V_{\lambda}$ de l'ouvert $U$ met en évidence une famille $\varphi_{\lambda}: U \longrightarrow \mathbb{R}$ $(\lambda \in U)$ de fonctions positives de classe $\mathcal{C}^{\infty}$ vérifiant :

i) $\operatorname{supp}\left(\varphi_{\lambda}\right) \subset V_{\lambda}$ pour tout $\lambda$ de $U$.

ii) Pour tout compact $K$ de $U$, un nombre fini seulement de $\varphi_{\lambda}$ ne sont pas identiquement nulles sur $K$.

iii) Pour tout $z \in U$ on a $\sum_{\lambda \in U} \varphi_{\lambda}(z)=1$.

On définit pour tout $\lambda \in U \quad u_{\lambda}^{\prime}: U \longrightarrow A$ et $v_{\lambda}^{\prime}, w_{\lambda}^{\prime}: U \longrightarrow \alpha$ par :

$$
\begin{gathered}
u_{\lambda}^{\prime}(z)=\varphi_{\lambda}(z) u_{\lambda}(z) \text { si } z \in V_{\lambda} \text { et } u_{\lambda}^{\prime}(z)=0 \text { sinon } \\
v_{\lambda}^{\prime}(z)=\varphi_{\lambda}(z) v_{\lambda}(z) \text { si } z \in V_{\lambda} \text { et } v_{\lambda}^{\prime}(z)=0 \text { sinon } \\
w_{\lambda}^{\prime}(z)=\varphi_{\lambda}(z) w_{\lambda}(z) \text { si } z \in V_{\lambda} \text { et } w_{\lambda}^{\prime}(z)=0 \text { sinon }
\end{gathered}
$$

$u_{\lambda}^{\prime}, v_{\lambda}^{\prime}, w_{\lambda}^{\prime}$ sont de classe $\mathcal{C}^{\infty}$ sur $U$. On pose :

$$
u(z)=\sum_{\lambda \in U} u_{\lambda}^{\prime}(z)
$$




$$
\begin{aligned}
v(z) & =\sum_{\lambda \in U} v_{\lambda}^{\prime}(z) \\
w(z) & =\sum_{\lambda \in U} w_{\lambda}^{\prime}(z)
\end{aligned}
$$

On a $u: U \longrightarrow A$ et $v, w: U \longrightarrow \alpha$ sont de classe $\mathcal{C}^{\infty}$ et vérifient :

$(a-z) u(z)+v(z)=u(z)(a-z)+w(z)=1$ pour tout $z \in U$.

D'où $(\tilde{a}-\tilde{\mathbf{z}}) \tilde{u}=\tilde{u}(\tilde{a}-\tilde{\mathbf{z}})=\tilde{1}$ dans $\mathcal{C}^{\infty}(U, A / \alpha)$.

\section{Remarque :}

Si $a^{\prime}$ est un autre représentant de $\tilde{a}$ et $u^{\prime}$ une autre application $u^{\prime}: U \longrightarrow A$ de classe $\mathcal{C}^{p}$ vérifiant $\left(\tilde{a}^{\prime}-\tilde{\mathbf{z}}\right) \tilde{u}^{\prime}=\tilde{u}^{\prime}\left(\tilde{a}^{\prime}-\tilde{\mathbf{z}}\right)=\tilde{1}$ alors $\tilde{u}=\tilde{u}^{\prime}$. Ceci découle de l'unicité de l'inverse dans une algèbre.

\section{Lemme :}

Soient $K$ un compact de $\mathbb{R}^{n}, U$ un voisinage ouvert de $K$ dans $\mathbb{R}^{n}(n \geq 1)$. Alors il existe une fonction $h: \mathbb{R}^{n} \longrightarrow[0,1]$ de classe $\mathcal{C}^{\infty}$ à support contenu dans $U$ et $h=1$ sur un voisinage de $K$.

Preuve :

Ceci découle du théorème d'existence d'une partition de l'unité.

\section{Proposition 3 :}

Soient $A / \alpha$ une algèbre de Banach quotient, $\tilde{a} \in A / \alpha, U$ un ouvert de $\mathbb{C}$ tel que : $U \supset \bar{B}\left(z_{o}, R\right) \supset B\left(z_{o}, R\right) \supset s p_{\alpha} a,\left(z_{o} \in U, R>0\right)$. Avec $B\left(z_{o}, R\right)$ la boule ouverte de centre $z_{o}$ et de rayon $R, \bar{B}\left(z_{o}, R\right)$ la boule fermée de centre $z_{o}$ et de rayon $R$. Alors il existe $h: \mathbb{C} \longrightarrow[0,1]$ de classe $\mathcal{C}^{\infty}$ à support contenu dans $B\left(z_{o}, R\right), h=1$ sur un voisinage de $s p_{\alpha} a$ et $u_{1}: \mathbb{C} \longrightarrow A$ de classe $\mathcal{C}^{\infty}$ tels que :

$(\tilde{a}-\tilde{\mathbf{z}}) \tilde{u}_{1}=\tilde{u}_{1}(\tilde{a}-\tilde{\mathbf{z}})=\tilde{1}-\tilde{h}$ dans $\mathcal{C}^{\infty}(\mathbb{C}, A / \alpha)$, où $\mathbf{z}$ désigne l'application qui à $z$ associe $z$.

Preuve :

Le lemme donne l'existence d'une telle application $h$. La proposition 2 donne l'existence de $u: \mathbb{C} \backslash s p_{\alpha} a \longrightarrow A$ et $v, w: \mathbb{C} \backslash s p_{\alpha} a \longrightarrow \alpha$ de classe $\mathcal{C}^{\infty}$ telles que :

$$
(a-z) u(z)+v(z)=u(z)(a-z)+w(z)=1 \text { pour tout } z \in \mathbb{C} \backslash s p_{\alpha} a .
$$

On définit $u_{1}: \mathbb{C} \longrightarrow A$ et $v_{1}, w_{1}: \mathbb{C} \longrightarrow \alpha$

par :

$$
\begin{aligned}
u_{1}(z) & =(1-h(z)) u(z) \text { si } z \in \mathbb{C} \backslash s p_{\alpha} a \text { et } u_{1}(z)=0 \text { si } z \in s p_{\alpha} a \\
v_{1}(z) & =(1-h(z)) v(z) \text { si } z \in \mathbb{C} \backslash s p_{\alpha} a \text { et } v_{1}(z)=0 \text { si } z \in s p_{\alpha} a \\
w_{1}(z) & =(1-h(z)) w(z) \text { si } z \in \mathbb{C} \backslash s p_{\alpha} a \text { et } w_{1}(z)=0 \text { si } z \in s p_{\alpha} a
\end{aligned}
$$

$u_{1}, v_{1}, w_{1}$ sont de classe $\mathcal{C}^{\infty}$ et on a :

$(a-z) u_{1}(z)+v_{1}(z)=u_{1}(z)(a-z)+w_{1}(z)=1-h(z)$ pour tout $z \in \mathbb{C}$. D'où $(\tilde{a}-\tilde{\mathbf{z}}) \tilde{u}_{1}=\tilde{u}_{1}(\tilde{a}-\tilde{\mathbf{z}})=\tilde{1}-\tilde{h} \operatorname{dans} \mathcal{C}^{\infty}(\mathbb{C}, A / \alpha)$. 


\subsection{Fonctions harmoniques}

Soient $A / \alpha$ une algèbre de Banach quotient, $U$ un ouvert de $\mathbb{C}$. Une application $f: U \longrightarrow A$ de classe $C^{\infty}$ est dite harmonique si le Laplacien $\triangle(f)=\frac{\partial^{2} f}{\partial x^{2}}+\frac{\partial^{2} f}{\partial y^{2}}$ est nul sur $U$.

\section{Remarque :}

Si $u$ est une forme linéaire continue de $A$ alors $\triangle(u o f)=u o \triangle(f)$. Comme le dual topologique de $A$ sépare les points de $A$, alors dire qu'une application $f$ de classe $\mathcal{C}^{\infty}$ est harmonique revient à dire que pour toute forme linéaire continue $u$ de $A$ uof est harmonique. On désigne par $h(U, A)$ l'espace vectoriel des applications $f: U \longrightarrow A$ harmoniques. $h(U, \alpha)$ est un sous espace vectoriel de $h(U, A)$. On pose $h(U, A / \alpha)=h(U, A) / h(U, \alpha)$. Si de plus $A$ est involutive et $\alpha$ est un idéal bilatère auto-adjoint de $A$ alors l'involution $h(U, A) \longrightarrow h(U, A)$ qui à $f$ associe $f^{*}$ envoie $h(U, \alpha)$ dans $h(U, \alpha)$, donc elle induit l'involution $h(U, A / \alpha) \longrightarrow h(U, A / \alpha)$ qui à $\tilde{f}$ associe $\tilde{f}^{*}$.

\section{Formule intégrale de Poisson :}

Soient $U$ un ouvert de $\mathbb{C}, z_{o} \in U, R>0$ tels que $\bar{B}\left(z_{o}, R\right) \subset U$. Alors pour toute application harmonique $f: U \longrightarrow A$ et pour tout $\left|z-z_{o}\right|<R$ on a :

$$
f(z)=\frac{1}{2 \pi i} \int_{\left|z^{\prime}-z_{o}\right|=R} f\left(z^{\prime}\right) \frac{R^{2}-\left|z-z_{o}\right|^{2}}{\left|z^{\prime}-z\right|^{2}} \frac{d z^{\prime}}{z^{\prime}-z_{o}} .
$$

(formule intégrale de Poisson)

Si on pose $z^{\prime}=z_{o}+R e^{i \theta}, 0 \leq \theta \leq 2 \pi$ alors on obtient

$$
f(z)=\frac{1}{2 \pi} \int_{0}^{2 \pi} f\left(z^{\prime}\right) \frac{R^{2}-\left|z-z_{o}\right|^{2}}{\left|z^{\prime}-z\right|^{2}} d \theta .
$$

\section{Remarque :}

La formule intégrale de Poisson pour les applications harmoniques à valeurs dans $A$ découle de la formule intégrale de Poisson pour les applications harmoniques à valeurs dans $\mathbb{C}$. Ceci découle du fait que le dual topologique de $A$ sépare les points de $A$.

\section{Calcul fonctionnel harmonique dans une algèbre de Banach involutive quotient}

Soient $A / \alpha$ une algèbre de Banach involutive quotient, $\tilde{a} \in A / \alpha, \mathrm{U}$ un ouvert de $\mathbb{C}$ tel que :

$$
s p_{\alpha} a \subset B\left(z_{0}, R\right) \subset \bar{B}\left(z_{o}, R\right) \subset U,\left(z_{o} \in U, R>0\right) .
$$

La proposition 2 de I met en évidence $u: \mathbb{C} \backslash s p_{\alpha} a \longrightarrow A$ de classe $\mathcal{C}^{\infty}$ tel que $\tilde{u}$ est l'inverse de $\tilde{a}-\tilde{\mathbf{z}}$ dans l'algèbre $\mathcal{C}^{\infty}\left(\mathbb{C} \backslash s p_{\alpha} a, A / \alpha\right)$, où $\mathbf{z}$ désigne l'application 
qui à z associe z.

\section{Définition :}

Sous les hypothèses précédentes, soit $\tilde{f}$ un élément de $h(U, A / \alpha)$.

On pose $\tilde{f}(\tilde{a})$ la classe d'équivalence modulo $\alpha$ de

$$
\frac{1}{2 \pi} \int_{0}^{2 \pi} f(z) u(z)\left[R^{2}-\left(a-z_{o}\right)\left(a-z_{o}\right)^{*}\right] u(z)^{*} d \theta,\left(z=z_{o}+R e^{i \theta}, 0 \leq \theta \leq 2 \pi\right) .
$$

\section{Remarques :}

1. $\tilde{f}(\tilde{a})$ a un sens car la fonction sous le signe intégrale est continue.

2. Si $\alpha=0$ alors $u(z)=(a-z)^{-1}$ pour tout $z \in \mathbb{C} \backslash s p_{\alpha} a$ et on retrouve le calcul fonctionnel harmonique dans une algébre de Banach involutive (cf A.ELKINANI [2])

\section{Proposition 4 :}

Sous les hypothèses précédentes, $\tilde{f}(\tilde{a})$ ne dépend pas du choix des représentants $a, u, f$.

Preuve:

Soient $a^{\prime}, u^{\prime}, f^{\prime}$ d'autres représentants respectivement de $\tilde{a}, \tilde{u}, \tilde{f}$.

On pose $\Omega=U \backslash s p_{\alpha} a$, alors $\tilde{a}=\tilde{a}^{\prime}, \tilde{u}=\tilde{u}^{\prime}, \tilde{f}=\tilde{f}^{\prime}$ dans l'algèbre $\mathcal{C}^{\infty}(\Omega, A / \alpha)$.

Donc $\tilde{f} \tilde{u}\left[\tilde{R}^{2}-\left(\tilde{a}-\tilde{z}_{o}\right)\left(\tilde{a}-\tilde{z}_{o}\right)^{*}\right] \tilde{u}^{*}=\tilde{f}^{\prime} \tilde{u}^{\prime}\left[\tilde{R}^{2}-\left(\tilde{a}^{\prime}-\tilde{z}_{o}\right)\left(\tilde{a}^{\prime}-\tilde{z}_{o}\right)^{*}\right] \tilde{u}^{\prime *}$ dans l'algèbre involutive $\mathcal{C}^{\infty}(\Omega, A / \alpha)$. Parsuite on a :

$$
\begin{aligned}
& \frac{1}{2 \pi} \int_{0}^{2 \pi} f(z) u(z)\left[R^{2}-\left(a-z_{o}\right)\left(a-z_{o}\right)^{*}\right] u(z)^{*} d \theta \text { est équivalent modulo } \alpha \text { à } \\
& \frac{1}{2 \pi} \int_{0}^{2 \pi} f^{\prime}(z) u^{\prime}(z)\left[R^{2}-\left(a^{\prime}-z_{o}\right)\left(a^{\prime}-z_{o}\right)^{*}\right] u^{\prime}(z)^{*} d \theta .
\end{aligned}
$$

\section{Théorème :}

Soient $A / \alpha$ une algèbre de Banach involutive quotient, $\tilde{a} \in A / \alpha, U$ un ouvert de $\mathbb{C}$ tel que $\operatorname{sp}_{\alpha} a \subset B\left(z_{o}, R\right) \subset \bar{B}\left(z_{o}, R\right) \subset U,\left(z_{o} \in U, R>0\right)$. Alors

i) l'application $h(U, A / \alpha) \longrightarrow A / \alpha$ qui à $\tilde{f}$ associe $\tilde{f}(\tilde{a})$ est linéaire.

ii) Si $f=1$ alors $\tilde{f}(\tilde{a})=\tilde{1}$.

iii) Si $f(z)=P(z)(a-z)$ avec $P$ un polynôme à coéfficients dans $A$ alors $\tilde{f}(\tilde{a})=\tilde{0}$.

iv) Si $f(z)=P(z)$ avec $P$ un polynôme à coéfficients dans $A$ alors $\tilde{f}(\tilde{a})=\tilde{P(} a)$.

\section{Preuve :}

i) Découle de la linéarité de l'intégrale.

ii) On a $f(z)=1$ donc $\tilde{f}(\tilde{a})$ est la classe d'équivalence modulo $\alpha$ de

$$
\frac{1}{2 \pi} \int_{0}^{2 \pi} u(z)\left[R^{2}-\left(a-z_{o}\right)\left(a-z_{o}\right)^{*}\right] u(z)^{*} d \theta .
$$

En utilisant le fait que $(\tilde{a}-\tilde{\mathbf{z}}) \tilde{u}=\tilde{u}(\tilde{a}-\tilde{\mathbf{z}})=\tilde{1}$ dans l'algèbre $\mathcal{C}^{\infty}\left(\mathbb{C} \backslash s p_{\alpha} a, A / \alpha\right)$ 
et en écrivant $a-z_{o}=(a-z)+\left(z-z_{o}\right)$ pour tout z de $\mathbb{C}$, on obtient $\tilde{f}(\tilde{a})$ est égal à la classe d'équivalence modulo $\alpha$ de

$$
-1-\frac{1}{2 \pi i} \int_{\left|z-z_{o}\right|=R} u(z) d z-\left[\frac{1}{2 \pi i} \int_{\left|z-z_{o}\right|=R} u(z) d z\right]^{*} .
$$
effet,

Pour conclure il suffit de prouver que $\frac{1}{2 \pi i} \int_{\left|z-z_{o}\right|=R} u(z) d z \equiv-1$ modulo $\alpha$. En

$$
\frac{1}{2 \pi i} \int_{\left|z-z_{o}\right|=R} u(z) d z=\frac{1}{2 \pi i} \int_{\left|z-z_{o}\right|=R} u_{1}(z) d z
$$

$u_{1}: \mathbb{C} \longrightarrow A$ est de classe $\mathcal{C}^{\infty}, u_{1}=u$ en dehors de $\operatorname{supp}(h)$ qui est contenu dans $B\left(z_{o}, R\right)$ (cf proposition3). On prend $R^{\prime}$ suffisament grand tel que $R^{\prime}>R$ et spa $\subset B\left(z_{o}, R^{\prime}\right)$. En utilisant la formule de Stokes on aura alors :

$$
\begin{aligned}
& \frac{1}{2 \pi i} \int_{\left|z-z_{o}\right|=R} u_{1}(z) d z=\frac{1}{2 \pi i} \int_{\left|z-z_{o}\right| \leq R} \frac{\partial u_{1}}{\partial \bar{z}}(z) d \bar{z} \wedge d z \\
& =\frac{1}{2 \pi i} \int_{\left|z-z_{o}\right| \leq R^{\prime}} \frac{\partial u_{1}}{\partial \bar{z}}(z) d \bar{z} \wedge d z-\frac{1}{2 \pi i} \int_{R<\left|z-z_{o}\right| \leq R^{\prime}} \frac{\partial u_{1}}{\partial \bar{z}}(z) d \bar{z} \wedge d z .
\end{aligned}
$$

On a $\frac{1}{2 \pi i} \int_{R<\left|z-z_{o}\right| \leq R^{\prime}} \frac{\partial u_{1}}{\partial \bar{z}}(z) d \bar{z} \wedge d z=\frac{1}{2 \pi i} \int_{R<\left|z-z_{o}\right| \leq R^{\prime}} \frac{\partial u}{\partial \bar{z}}(z) d \bar{z} \wedge d z$.

Or $(\tilde{a}-\tilde{\mathbf{z}}) \tilde{u}=\tilde{u}(\tilde{a}-\tilde{\mathbf{z}})=\tilde{1}$ dans l'algèbre $\mathcal{C}^{\infty}\left(\mathbb{C} \backslash s p_{\alpha} a, A / \alpha\right)$ alors

$\frac{\partial u}{\partial \bar{z}}: \mathbb{C} \backslash s p_{\alpha} a: \longrightarrow \alpha$ est de classe $\mathcal{C}^{\infty}$ donc

$\frac{1}{2 \pi i} \int_{\left|z-z_{o}\right|=R} u(z) d z \equiv \frac{1}{2 \pi i} \int_{\left|z-z_{o}\right| \leq R^{\prime}} \frac{\partial u_{1}}{\partial \bar{z}}(z) d \bar{z} \wedge d z$ modulo $\alpha$.

La formule de Stokes donne

$$
\begin{aligned}
& \frac{1}{2 \pi i} \int_{\left|z-z_{o}\right| \leq R^{\prime}} \frac{\partial u_{1}}{\partial \bar{z}}(z) d \bar{z} \wedge d z=\frac{1}{2 \pi i} \int_{\left|z-z_{o}\right|=R^{\prime}} u_{1}(z) d z \\
& =\frac{1}{2 \pi i} \int_{\left|z-z_{o}\right|=R^{\prime}} u(z) d z \equiv \frac{1}{2 \pi i} \int_{\left|z-z_{o}\right|=R^{\prime}}(a-z)^{-1} d z \text { modulo } \alpha . \\
& \text { Or } \frac{1}{2 \pi i} \int_{\left|z-z_{o}\right|=R^{\prime}}(a-z)^{-1} d z=-1, \text { d'où le résultat cherché. }
\end{aligned}
$$

iii) Soit $f(z)=P(z)(a-z)$ où $P$ est un polynôme à coéfficients dans $A$. On se ramène au cas $f(z)=\left(z-z_{o}\right)^{n}(a-z), n \geq 0$. $\tilde{f}(\tilde{a})$ est la classe d'équivalence modulo $\alpha$ de

$$
\frac{1}{2 \pi} \int_{0}^{2 \pi}\left(z-z_{o}\right)^{n}(a-z) u(z)\left[R^{2}-\left(a-z_{o}\right)\left(a-z_{o}\right)^{*}\right] u(z)^{*} d \theta \text {. Or }(\tilde{a}-\tilde{\mathbf{z}}) \tilde{u}=\tilde{u}(\tilde{a}-\tilde{\mathbf{z}})=
$$
$\tilde{1}$ dans l'algèbre $\mathcal{C}^{\infty}\left(\mathbb{C} \backslash s p_{\alpha} a, A / \alpha\right)$, donc

$$
\frac{1}{2 \pi} \int_{0}^{2 \pi}\left(z-z_{o}\right)^{n}(a-z) u(z)\left[R^{2}-\left(a-z_{o}\right)\left(a-z_{o}\right)^{*}\right] u(z)^{*} d \theta
$$

$\equiv \frac{\left[R^{2}-\left(a-z_{o}\right)\left(a-z_{o}\right)^{*}\right]}{2 \pi} \int_{0}^{2 \pi}\left(z-z_{o}\right)^{n} u(z)^{*} d \theta$. Tout revient à prouver que $\int_{0}^{2 \pi}\left(z-z_{o}\right)^{n} u(z)^{*} d \theta \in \alpha$, ou encore $\int_{0}^{2 \pi}\left(\overline{z-z}_{o}\right)^{n} u(z) d \theta \in \alpha$, puisque $\alpha$ est auto-adjoint et l' involution * est continue.

$$
\begin{aligned}
& \int_{0}^{2 \pi}\left(\overline{z-z_{o}}\right)^{n} u(z) d \theta=R^{2 n} \int_{0}^{2 \pi} \frac{u(z)}{\left(z-z_{o}\right)^{n}} d \theta\left(\operatorname{car}\left|z-z_{o}\right|=R\right) \\
& =\frac{R^{2 n}}{i} \int_{\left|z-z_{o}\right|=R} \frac{u(z)}{\left(z-z_{o}\right)^{n+1}} d z \text {. Montrons alors que } \int_{\left|z-z_{o}\right|=R} \frac{u(z)}{\left(z-z_{o}\right)^{n+1}} d z \in \alpha .
\end{aligned}
$$

En effet, il existe $k: \mathbb{C} \longrightarrow[0,1]$ de classe $\mathcal{C}^{\infty}$ telle que $k=1$ sur un voisinage de $z_{\mathrm{o}}$ et $\operatorname{supp}(k) \subset B\left(z_{o}, R\right)$. On pose : 
$\varphi: \mathbb{C} \longrightarrow \mathbb{C}$ définie par $\varphi(z)=\frac{1-k(z)}{\left(z-z_{o}\right)^{n+1}}$ si $z \neq z_{o}$ et $\varphi(z)=0$ si $z=z_{o} . \varphi$ est de classe $\mathcal{C}^{\infty}$ et $\varphi(z)=\frac{1}{\left(z-z_{o}\right)^{n+1}}$ en dehors de $\operatorname{supp}(k)$.

$\int_{\left|z-z_{o}\right|=R} \frac{u(z)}{\left(z-z_{o}\right)^{n+1}} d z=\int_{\left|z-z_{o}\right|=R} \varphi(z) u_{1}(z) d z$

$=\int_{\left|z-z_{o}\right| \leq R} \frac{\partial\left(\varphi u_{1}\right)}{\partial \bar{z}}(z) d \bar{z} \wedge d z$ (formule de Stokes).

On prend $R^{\prime}$ suffisament grands de sorte que $R^{\prime}>R$ et $R^{\prime}>\left\|a-z_{o}\right\|$. Donc

$\int_{\left|z-z_{o}\right| \leq R} \frac{\partial\left(\varphi u_{1}\right)}{\partial \bar{z}}(z) d \bar{z} \wedge d z$

$=\int_{\left|z-z_{o}\right| \leq R^{\prime}} \frac{\partial\left(\varphi u_{1}\right)}{\partial \bar{z}}(z) d \bar{z} \wedge d z-\int_{R<\left|z-z_{o}\right| \leq R^{\prime}} \frac{\partial\left(\varphi u_{1}\right)}{\partial \bar{z}}(z) d \bar{z} \wedge d z$

On a $\frac{\partial\left(\varphi u_{1}\right)}{\partial \bar{z}}:\left\{z|| z-z_{o} \mid>R\right\} \longrightarrow \alpha$ est de classe $\mathcal{C}^{\infty}$. Donc

$\int_{\left|z-z_{o}\right| \leq R} \frac{\partial\left(\varphi u_{1}\right)}{\partial \bar{z}}(z) d \bar{z} \wedge d z$

$\equiv \int_{\left|z-z_{o}\right| \leq R^{\prime}} \frac{\partial\left(\varphi u_{1}\right)}{\partial \bar{z}}(z) d \bar{z} \wedge d z$ modulo $\alpha$

$=\int_{\left|z-z_{o}\right|=R^{\prime}}\left(\varphi u_{1}\right)(z) d z \equiv \int_{\left|z-z_{o}\right|=R^{\prime}} \frac{(a-z)^{-1}}{\left(z-z_{o}\right)^{n+1}} d z$ modulo $\alpha$.

En écrivant $z-a=\left(z-z_{o}\right)-\left(a-z_{o}\right)$ et en utilisant le fait que $\left\|\frac{a-z_{o}}{z-z_{o}}\right\|=$ $\frac{\left\|a-z_{o}\right\|}{R^{\prime}}<1$ on aura $\int_{\left|z-z_{o}\right|=R^{\prime}} \frac{(a-z)^{-1}}{\left(z-z_{o}\right)^{n+1}} d z=-i \sum_{k=0}^{+\infty}\left(a-z_{o}\right)^{k} \int_{0}^{2 \pi}\left(z-z_{o}\right)^{-n-k-1} d \theta$. $\alpha$.

Or $\int_{0}^{2 \pi}\left(z-z_{o}\right)^{-n-k-1} d \theta=0, \forall k \geq 0$, donc $\int_{\left|z-z_{o}\right|=R} \frac{u(z)}{\left(z-z_{o}\right)^{n+1}} d z \equiv 0$ modulo

iv) Soit $f(z)=P(z)$ avec $P$ un polynôme à coéfficients dans $A$.

On pose : $g(z)=f(z)-P(a)$. Alors $g(z)=Q(z)(a-z)$ avec $Q$ un polynôme à coéfficients dans $A$. Le iii) achève la démonstration.

\section{Proposition 5 :}

Sous les hypothèses du théorème 1 on suppose de plus que l'algèbre $A$ est commutative, que l'algèbre $A / \alpha$ est hermitienne et que $f$ est harmonique à valeurs dans $\mathbb{C}$. Soit $\chi^{\prime}$ un caractère de l'algèbre $A / \alpha$. Alors $\chi^{\prime}(\tilde{f}(\tilde{a}))=f\left(\chi^{\prime}(\tilde{a})\right)$.

Preuve :

Soit $\chi^{\prime}$ un caractère de l'algèbre $A / \alpha$.

$\chi: A \longrightarrow \mathbb{C}$

$a \longrightarrow \chi(a)=\chi^{\prime}(\tilde{a})$ est un caractère de $A$ dont le noyau contient $\alpha$. Comme $\chi^{\prime}$ est hermitien alors $\chi$ l'est aussi.

$\chi^{\prime}(\tilde{f}(\tilde{a}))=\chi\left[\frac{1}{2 \pi} \int_{0}^{2 \pi} f(z) u(z)\left[R^{2}-\left(a-z_{o}\right)\left(a-z_{o}\right)^{*}\right] u(z)^{*} d \theta\right]$.

Comme $\chi$ est continu et hermitien alors 


$$
\left.\chi^{\prime}(\tilde{f}(\tilde{a}))=\frac{1}{2 \pi} \int_{0}^{2 \pi} f(z) \chi(u(z))\left[R^{2}-\left(\chi(a)-z_{o}\right)\left(\overline{\chi(a)-z_{o}}\right)\right] \overline{\chi(u(z)}\right) d \theta
$$

$\operatorname{Or}(a-z) u(z)+v(z)=1, v(z) \in \alpha(\operatorname{cf}$ proposition 2). Comme $\alpha \subset \operatorname{Ker}(\chi)$, alors $(\chi(a)-z) \chi(u(z))=1$.

D'où $\chi^{\prime}(\tilde{f}(\tilde{a}))=\frac{1}{2 \pi} \int_{0}^{2 \pi} f(z) \frac{R^{2}-\left(\chi(a)-z_{o}\right)\left(\overline{\chi(a)-z_{o}}\right)}{|\chi(a)-z|^{2}} d \theta=f(\chi(a))=f\left(\chi^{\prime}(\tilde{a})\right)$.

\section{Proposition 6 :}

Soient $A / \alpha$ une algèbre de Banach involutive quotient, $\tilde{a} \in A / \alpha, U$ un ouvert de $\mathbb{C}$ tel que $\operatorname{sp}_{\alpha} a \subset B\left(z_{o}, R\right) \subset \bar{B}\left(z_{o}, R\right) \subset U, \tilde{f}$ un élément de $h(U, A / \alpha)$. Si $\tilde{a}$ commute avec $\tilde{f}$ et $\tilde{f}^{*}$ dans l'algèbre $\mathcal{C}^{\infty}(U, A / \alpha)$ alors $\tilde{f}^{*}(\tilde{a})=(\tilde{f}(\tilde{a}))^{*}$.

Preuve:

$\tilde{f}^{*}(\tilde{a})$ est la classe d'équivalence modulo $\alpha$ de

$\frac{1}{2 \pi} \int_{0}^{2 \pi} f^{*}(z) u(z)\left[R^{2}-\left(a-z_{o}\right)\left(a-z_{o}\right)^{*}\right] u(z)^{*} d \theta$

$=\left[\frac{1}{2 \pi} \int_{0}^{2 \pi} u(z)\left[R^{2}-\left(a-z_{o}\right)\left(a-z_{o}\right)^{*}\right] u(z)^{*} f(z) d \theta\right]^{*}$ car l'involution est continue.

On pose : $\Omega=U \backslash s p_{\alpha} a$. $\tilde{u}$ est l'inverse de $\tilde{a}-\tilde{\mathbf{z}}$ dans l'algèbre $\mathcal{C}^{\infty}(\Omega, A / \alpha)$. $\tilde{f}$ et $\tilde{f}^{*}$ commutent avec $\tilde{a}-\tilde{\mathbf{z}}$ dans l'algèbre $\mathcal{C}^{\infty}(\Omega, A / \alpha)$ donc $\tilde{f}$ et $\tilde{f}^{*}$ commutent avec $\tilde{u}$ dans $\mathcal{C}^{\infty}(\Omega, A / \alpha)$. Parsuite $\tilde{f}$ commute avec $\tilde{a}, \tilde{a}^{*}, \tilde{u}, \tilde{u}^{*}$ dans $\mathcal{C}^{\infty}(\Omega, A / \alpha)$.

Alors $\tilde{u}\left[\tilde{R}^{2}-\left(\tilde{a}-\tilde{z}_{o}\right)\left(\tilde{a}-\tilde{z}_{o}\right)^{*}\right] \tilde{u}^{*} \tilde{f}=\tilde{f} \tilde{u}\left[\tilde{R}^{2}-\left(\tilde{a}-\tilde{z}_{o}\right)\left(\tilde{a}-\tilde{z}_{o}\right)^{*}\right] \tilde{u}^{*} \operatorname{dans} \mathcal{C}^{\infty}(\Omega, A / \alpha)$. Donc

$$
\begin{aligned}
& \frac{1}{2 \pi} \int_{0}^{2 \pi} u(z)\left[R^{2}-\left(a-z_{o}\right)\left(a-z_{o}\right)^{*}\right] u(z)^{*} f(z) d \theta \\
& \equiv \frac{1}{2 \pi} \int_{0}^{2 \pi} f(z) u(z)\left[R^{2}-\left(a-z_{o}\right)(a-z)^{*}\right] u(z)^{*} d \theta \text { modulo } \alpha . \\
& \text { D'où } \tilde{f}^{*}(\tilde{a})=(\tilde{f}(\tilde{a}))^{*} .
\end{aligned}
$$

\section{Proposition 7 :}

Soient $A / \alpha$ une algèbre de Banach involutive quotient, $\tilde{a} \in A / \alpha$ et $U$ un ouvert de $\mathbb{C}$ tel que $\operatorname{sp}_{\alpha} a \subset B\left(z_{o}, R\right) \subset \bar{B}\left(z_{o}, R\right) \subset U,\left(z_{o} \in U, R>0\right)$. Soit $f: U \longrightarrow A$ holomorphe. Alors $\tilde{f}(\tilde{a})$ coincide avec $f[a]$ du calcul fonctionnel holomorphe défini par L.WAELBROECK [6].

\section{Preuve:}

Il existe $R^{\prime}>R$ tel que $\bar{B}\left(z_{o}, R^{\prime}\right) \subset U . \tilde{f}(\tilde{a})$ est la classe d'équivalence modulo $\alpha \operatorname{de} \frac{1}{2 \pi} \int_{0}^{2 \pi} f(z) u(z)\left[R^{2}-\left(a-z_{o}\right)\left(a-z_{o}\right)^{*}\right] u(z)^{*} d \theta$.

Comme $f$ est holomorphe alors $f(z)=\frac{1}{2 \pi i} \int_{\left|z^{\prime}-z_{o}\right|=R^{\prime}} \frac{f\left(z^{\prime}\right)}{z^{\prime}-z} d z^{\prime}$. Donc $\frac{1}{2 \pi} \int_{0}^{2 \pi} f(z) u(z)\left[R^{2}-\left(a-z_{o}\right)\left(a-z_{o}\right)^{*}\right] u(z)^{*} d \theta=\frac{1}{2 \pi i} \int_{\left|z^{\prime}-z_{o}\right|=R^{\prime}} f\left(z^{\prime}\right) g\left(z^{\prime}\right) d z^{\prime}$, $\operatorname{avec} g\left(z^{\prime}\right)=\frac{1}{2 \pi} \int_{0}^{2 \pi} \frac{1}{z^{\prime}-z} u(z)\left[R^{2}-\left(a-z_{o}\right)\left(a-z_{o}\right)^{*}\right] u(z)^{*} d \theta$. 
Montrons que $z^{\prime} \longrightarrow\left(z^{\prime}-a\right) g\left(z^{\prime}\right)-1$ est de classe $\mathcal{C}^{\infty}$ sur $\left\{z^{\prime}|| z^{\prime}-z_{o} \mid>R\right\}$ à valeurs dans $\alpha$. En effet, $\frac{z^{\prime}-a}{z^{\prime}-z}=\frac{\left(z^{\prime}-z\right)-(a-z)}{z^{\prime}-z}=1-\frac{a-z}{z^{\prime}-z}$, donc

$$
\left(z^{\prime}-a\right) g\left(z^{\prime}\right)=1+x-\frac{1}{2 \pi} \int_{0}^{2 \pi} \frac{a-z}{z^{\prime}-z} u(z)\left[R^{2}-\left(a-z_{o}\right)\left(a-z_{o}\right)^{*}\right] u(z)^{*} d \theta \text { avec } x
$$

est une constant de $\alpha$. Tout revient à prouver que l'application qui à $z^{\prime}$ associe $\frac{1}{2 \pi} \int_{0}^{2 \pi} \frac{a-z}{z^{\prime}-z} u(z)\left[R^{2}-\left(a-z_{o}\right)\left(a-z_{o}\right)^{*}\right] u(z)^{*} d \theta$ est de classe $\mathcal{C}^{\infty}$ sur $\left\{z^{\prime}|| z^{\prime}-z_{o} \mid>R\right\}$ à valeurs dans $\alpha$. On a $(a-z) u(z)=1-v(z)$ pour tout $z \in \mathbb{C} \backslash s p_{\alpha} a$ avec $v$ de classe $\mathcal{C}^{\infty}$ à valeurs dans $\alpha(\operatorname{cf}$ proposition 2$)$. Donc

$\frac{1}{2 \pi} \int_{0}^{2 \pi} \frac{a-z}{z^{\prime}-z} u(z)\left[R^{2}-\left(a-z_{o}\right)\left(a-z_{o}\right)^{*}\right] u(z)^{*} d \theta=$

$$
\frac{1}{2 \pi}\left[R^{2}-\left(a-z_{o}\right)\left(a-z_{o}\right)^{*}\right] g_{1}\left(z^{\prime}\right)-g_{2}\left(z^{\prime}\right)
$$

avec $g_{1}\left(z^{\prime}\right)=\int_{0}^{2 \pi} \frac{u(z)^{*}}{z^{\prime}-z} d \theta$ et $g_{2}\left(z^{\prime}\right)=\frac{1}{2 \pi} \int_{0}^{2 \pi} \frac{1}{z^{\prime}-z} v(z)\left[R^{2}-\left(a-z_{o}\right)\left(a-z_{o}\right)^{*}\right] u(z)^{*} d \theta$.

$g_{2}$ est holomorphe sur $\left\{z^{\prime} \in \mathbb{C}|| z^{\prime}-z_{o} \mid>R\right\}$ à valeurs dans $\alpha$, donc elle est de classe $\mathcal{C}^{\infty}$ à valeurs dans $\alpha$. Pour conclure il suffit de prouver que $g_{1}$ est de classe $\mathcal{C}^{\infty}$ à valeurs dans $\alpha$.

$$
g_{1}\left(z^{\prime}\right)=\left[\int_{0}^{2 \pi} \frac{u(z)}{\overline{z^{\prime}-z}} d \theta\right]^{*} \text {, tout revient alors à prouver que } z^{\prime} \longrightarrow \int_{0}^{2 \pi} \frac{u(z)}{\overline{z^{\prime}-z}} d \theta
$$

est de classe $\mathcal{C}^{\infty}$ sur $\left\{z^{\prime} \in \mathbb{C}|| z^{\prime}-z_{o} \mid>R\right\}$ à valeurs dans $\alpha$.

$$
\begin{aligned}
& \int_{0}^{2 \pi} \frac{u(z)}{\overline{z^{\prime}-z}} d \theta=\int_{0}^{2 \pi} \frac{1}{\left(\overline{z^{\prime}-z_{o}}\right)} \frac{1}{1-\frac{\left(\overline{z-z_{o}}\right)}{\left(\overline{z^{\prime}-z_{o}}\right)}} u(z) d \theta \\
& =\int_{0}^{2 \pi} \sum_{n=0}^{+\infty} \frac{\left(\overline{z-z_{o}}\right)^{n}}{\left(\overline{z^{\prime}-z_{o}}\right)^{n+1}} u(z) d \theta \text { la convergrence est uniforme donc } \\
& =\sum_{n=0}^{+\infty}\left[\frac{1}{\left(\overline{z^{\prime}-z_{o}}\right)^{n+1}} \int_{0}^{2 \pi}\left(\overline{z-z_{o}}\right)^{n} u(z) d \theta\right], \text { or }\left|z-z_{o}\right|=R, \text { alors } \\
& \int_{0}^{2 \pi} \frac{u(z)}{\overline{z^{\prime}-z}} d \theta=\sum_{n=0}^{+\infty}\left[\frac{R^{2 n}}{\left(\overline{z^{\prime}-z_{o}}\right)^{n+1}} \int_{0}^{2 \pi} \frac{u(z)}{\left(z-z_{o}\right)^{n}} d \theta\right] \\
& =\sum_{n=0}^{+\infty}\left[\frac{R^{2 n}}{i\left(\overline{z^{\prime}-z_{o}}\right)^{n+1}} \int_{\left|z-z_{o}\right|=R} \frac{u(z)}{\left(z-z_{o}\right)^{n+1}} d z\right]
\end{aligned}
$$

Il existe $k: \mathbb{C} \longrightarrow[0,1]$ de classe $\mathcal{C}^{\infty}$ telle que $k=1$ sur un voisinage de $z_{\mathrm{o}}$ et $\operatorname{supp}(k) \subset B\left(z_{o}, R\right)$.

On pose $: \varphi_{n}(z)=\frac{1-k(z)}{\left(z-z_{0}\right)^{n+1}}$ si $z \neq z_{o}$ et $\varphi_{n}(z)=0$ si $z=z_{o}$. Donc

$$
\begin{aligned}
& \int_{0}^{2 \pi} \frac{u(z)}{\overline{z^{\prime}-z}} d \theta=\sum_{n=0}^{+\infty}\left[\frac{R^{2 n}}{i\left(\overline{z^{\prime}-z_{o}}\right)^{n+1}} \int_{\left|z-z_{o}\right|=R}\left(\varphi_{n} u_{1}\right)(z) d z\right. \\
& =\sum_{n=0}^{+\infty}\left[\frac{R^{2 n}}{i\left(\overline{z^{\prime}-z_{o}}\right)^{n+1}} \int_{\left|z-z_{o}\right| \leq R} \frac{\partial}{\partial \bar{z}}\left(\varphi_{n} u_{1}\right)(z) d \bar{z} \wedge d z\right] \text { (formule de Stokes) } \\
& =\sum_{n=0}^{+\infty}\left[\frac{R^{2 n}}{i\left(\overline{z^{\prime}-z_{o}}\right)^{n+1}} \int_{\left|z-z_{o}\right| \leq R^{\prime \prime}} \frac{\partial}{\partial \bar{z}}\left(\varphi_{n} u_{1}\right)(z) d \bar{z} \wedge d z\right] \\
& -\sum_{n=0}^{+\infty}\left[\frac{R^{2 n}}{i\left(\overline{z^{\prime}-z_{o}}\right)^{n+1}} \int_{R<\left|z-z_{o}\right| \leq R^{\prime \prime}} \frac{\partial}{\partial \bar{z}}\left(\varphi_{n} u_{1}\right)(z) d \bar{z} \wedge d z\right]
\end{aligned}
$$


avec $R^{\prime \prime}$ suffisament grand tel que $R^{\prime \prime}>R^{\prime}>R$ et $R^{\prime \prime}>\left\|a-z_{o}\right\|$.

Pour tout $\left|z-z_{o}\right|>R$ on a $\frac{\partial}{\partial \bar{z}}\left(\varphi_{n} u_{1}\right)(z)=\frac{1}{\left(z-z_{o}\right)^{n+1}} \frac{\partial}{\partial \bar{z}} u(z)$ et $\frac{\partial}{\partial \bar{z}} u$ est de classe $\mathcal{C}^{\infty}$ sur $\left\{z \in \mathbb{C}|| z-z_{o} \mid>R\right\}$ à valeurs dans $\alpha$.

$\sum_{n=0}^{+\infty}\left[\frac{R^{2 n}}{i\left(\overline{z^{\prime}-z_{o}}\right)^{n+1}} \int_{R<\left|z-z_{o}\right| \leq R^{\prime \prime}} \frac{\partial}{\partial \bar{z}}\left(\varphi_{n} u_{1}\right)(z) d \bar{z} \wedge d z\right]$
$=\sum_{n=0}^{+\infty}\left[\frac{R^{2 n}}{i\left(\overline{z^{\prime}-z_{o}}\right)^{n+1}} \int_{R<\left|z-z_{o}\right| \leq R^{\prime \prime}} \frac{1}{\left(z-z_{o}\right)^{n+1}} \frac{\partial}{\partial \bar{z}} u(z) d \bar{z} \wedge d z\right]$ cette fonction en $z^{\prime}$ étant le conjugué (pour l'involution *) d'une application holomorphe sur $\left\{z^{\prime} \in \mathbb{C}|| z^{\prime}-z_{o} \mid>R\right\}$ à valeurs dans $\alpha$.

D'autre part on a :

$\sum_{n=0}^{+\infty}\left[\frac{R^{2 n}}{i\left(\overline{z^{\prime}-z_{o}}\right)^{n+1}} \int_{\left|z-z_{o}\right| \leq R^{\prime \prime}} \frac{\partial}{\partial \bar{z}}\left(\varphi_{n} u_{1}\right)(z) d \bar{z} \wedge d z\right]$

$=\sum_{n=0}^{+\infty}\left[\frac{R^{2 n}}{i\left(\overline{z^{\prime}-z_{o}}\right)^{n+1}} \int_{\left|z-z_{o}\right|=R^{\prime \prime}}\left(\varphi_{n} u_{1}\right)(z) d z\right]$ (formule de Stokes)

$=\sum_{n=0}^{+\infty}\left[\frac{R^{2 n}}{i\left(\overline{z^{\prime}-z_{o}}\right)^{n+1}} \int_{\left|z-z_{o}\right|=R^{\prime \prime}}\left(\varphi_{n} u\right)(z) d z\right]$

$=\sum_{n=0}^{+\infty}\left[\frac{R^{2 n}}{i\left(\overline{z^{\prime}-z_{o}}\right)^{n+1}} \int_{\left|z-z_{o}\right|=R^{\prime \prime}} \frac{1}{\left(z-z_{o}\right)^{n+1}}(a-z)^{-1} d z\right]-g_{3}\left(z^{\prime}\right)$ avec

$g_{3}\left(z^{\prime}\right)=\sum_{n=0}^{+\infty}\left[\frac{R^{2 n}}{i\left(\overline{z^{\prime}-z_{o}}\right)^{n+1}} \int_{\left|z-z_{o}\right|=R^{\prime \prime}} \frac{1}{\left(z-z_{o}\right)^{n+1}}(a-z)^{-1} v(z) d z\right]$.

Or $\int_{\left|z-z_{o}\right|=R^{\prime \prime}} \frac{1}{\left(z-z_{o}\right)^{n+1}}(a-z)^{-1} d z$

$=-\int_{\left|z-z_{o}\right|=R^{\prime \prime}} \frac{1}{\left(z-z_{o}\right)^{n+1}}\left(\left(z-z_{o}\right)-\left(a-z_{o}\right)\right)^{-1} d z$. Comme $\left\|a-z_{o}\right\|<\left|z-z_{o}\right|=$ $R^{\prime \prime}$, on aura

$=-\int_{\left|z-z_{o}\right|=R^{\prime \prime}} \sum_{k=0}^{+\infty} \frac{\left(a-z_{o}\right)^{k}}{\left(z-z_{o}\right)^{n+k+2}} d z$, la convergence est uniforme donc

$\int_{\left|z-z_{o}\right|=R^{\prime \prime}} \frac{1}{\left(z-z_{o}\right)^{n+1}}(a-z)^{-1} d z=-\sum_{k=0}^{+\infty}\left(a-z_{o}\right)^{k} \int_{\left|z-z_{o}\right|=R^{\prime \prime}} \frac{d z}{\left(z-z_{o}\right)^{n+k+2}}$

Or $\int_{\left|z-z_{o}\right|=R^{\prime \prime}} \frac{d z}{\left(z-z_{o}\right)^{n+k+2}}=0$ pour tout $n \geq 0$ et pour tout $k \geq 0$ ), donc

$\int_{\left|z-z_{o}\right|=R^{\prime \prime}} \frac{1}{\left(z-z_{o}\right)^{n+1}}(a-z)^{-1} d z=0$ pour tout $n \geq 0$ d'où

$\sum_{n=0}^{+\infty}\left[\frac{R^{2 n}}{i\left(\overline{z^{\prime}-z_{o}}\right)^{n+1}} \int_{\left|z-z_{o}\right| \leq R^{\prime \prime}} \frac{\partial}{\partial \bar{z}}\left(\varphi_{n} u_{1}\right)(z) d \bar{z} \wedge d z\right]=0$ pour tout $z$ vérifiant $\mid z-$ $z_{o} \mid>R$.

$g_{3}$ est le conjugué (pour l'involution *) d'une application holomorphe à valeurs dans $\alpha$.

Conclusion : $z^{\prime} \longrightarrow\left(z^{\prime}-a\right) g\left(z^{\prime}\right)-1$ est de classe $\mathcal{C}^{\infty}$ sur $\left\{z^{\prime} \in \mathbb{C}|| z^{\prime}-z_{o} \mid>R\right\}$ à valeurs dans $\alpha$.

Donc $z^{\prime} \longrightarrow g\left(z^{\prime}\right)+u\left(z^{\prime}\right)$ est de classe $\mathcal{C}^{\infty}$ sur $\left\{z^{\prime} \in \mathbb{C}|| z^{\prime}-z_{o} \mid>R\right\}$ à valeurs dans $\alpha$. 
D'où $\tilde{f}(\tilde{a})$ est la classe d'équivalence modulo $\alpha$ de

$$
\begin{aligned}
& -\frac{1}{2 \pi i} \int_{\left|z^{\prime}-z_{o}\right|=R^{\prime}} f\left(z^{\prime}\right) u\left(z^{\prime}\right) d z^{\prime}=-\frac{1}{2 \pi i} \int_{\left|z^{\prime}-z_{o}\right|=R^{\prime}} f\left(z^{\prime}\right) u_{1}\left(z^{\prime}\right) d z^{\prime} \\
& \equiv-\frac{1}{2 \pi i} \int_{\left|z^{\prime}-z_{o}\right| \leq R^{\prime}} f\left(z^{\prime}\right) 2 h \frac{\partial u_{1}}{\partial \bar{z}^{\prime}} d \bar{z}^{\prime} \wedge d z^{\prime} \text { modulo } \alpha . \\
& \text { D'où } \tilde{f}(\tilde{a})=f[a] .
\end{aligned}
$$

Proposition 8 : (théorème de J.W.M.FORD dans une algèbre de Banach involutive quotient [3], voir aussi le cas $\alpha=0$ de A.ELKINANI [2]).

Soient $A / \alpha$ une algèbre de Banach involutive quotient, $\tilde{a}$ un élément hermitien de $A / \alpha$ tel que $\operatorname{sp}_{\alpha} a \subset\{z \in \mathbb{C} \mid \operatorname{Re}(z)>0\}$. Alors il existe un élément hermitien $\tilde{b}$ de $A / \alpha$ tel que $\tilde{b}^{2}=\tilde{a}$.

\section{Preuve :}

On pose : $U=\{z \in \mathbb{C} \mid \operatorname{Re}(z)>0\} \mathrm{U}$ est un ouvert de $\mathbb{C}$ contenant $s p_{\alpha} a$. Il existe $z_{o}>0, R>0$ tels que $s p_{\alpha} a \subset B\left(z_{o}, R\right) \subset \bar{B}\left(z_{o}, R\right) \subset U$. Il existe une fonction $f$ holomorphe sur $U$ telle que $f^{2}(z)=z$ pour tout $z \in U$ et $f(1)=1$. On pose : $\tilde{b}=f[a]$ définit par le calcul fonctionnel holomorphe (cf L.WAELBROECK [6]). Comme $f^{2}(z)=z$ pour tout $z \in U$ alors $\tilde{b}^{2}=\tilde{a}$. Il reste à prouver que $\tilde{b}$ est hermitien . D'après (la proposition 7) on a $\tilde{b}=f[a]=\tilde{f}(\tilde{a})$, donc $\tilde{b}^{*}$ est la classe d'équivalence modulo $\alpha$ de

$$
\begin{aligned}
& \frac{1}{2 \pi} \int_{0}^{2 \pi} \overline{f(z)} u(z)\left[R^{2}-\left(a-z_{o}\right)\left(a-z_{o}\right)^{*}\right] u(z)^{*} d \theta,\left(z=z_{o}+R e^{i \theta}\right) \\
& =\frac{1}{2 \pi} \int_{0}^{2 \pi} f(\bar{z}) u(z)\left[R^{2}-\left(a-z_{o}\right)\left(a-z_{o}\right)^{*}\right] u(z)^{*} d \theta \operatorname{car} \overline{f(z)}=f(\bar{z})
\end{aligned}
$$

Il existe $0<R^{\prime}<R$ tel que $s p_{\alpha} a \subset B\left(z_{o}, R^{\prime}\right)$, on pose : $\Omega=\{z \in \mathbb{C} \mid \operatorname{Re}(z)>$ Det $\left.\left|z-z_{o}\right|>R^{\prime}\right\}$.

$\Omega$ est un ouvert de $\mathbb{C}$ invariant par l'application $z \longmapsto \bar{z}$.

On a : $u(z)(a-z)+v(z)=(a-z) u(z)+w(z)=1$ pour tout $z \in \Omega$ donc $u(\bar{z})(a-\bar{z})+v(\bar{z})=(a-\bar{z}) u(\bar{z})+w(\bar{z})=1$ pour tout $z \in \Omega$. Ceci donne $z \longrightarrow u(z)-u(\bar{z})^{*}$ et $z \longrightarrow u(z)^{*}-u(\bar{z})$ sont de classe $\mathcal{C}^{\infty}$ sur $\Omega$ à valeurs dans $\alpha$. Donc $\tilde{b}^{*}$ est la classe d'équivalence modulo $\alpha$ de

$$
\begin{aligned}
& \frac{1}{2 \pi} \int_{0}^{2 \pi} f(\bar{z}) u(\bar{z})^{*}\left[R^{2}-\left(a-z_{o}\right)\left(a-z_{o}\right)^{*}\right] u(\bar{z}) d \theta \\
& =\frac{1}{2 \pi} \int_{0}^{2 \pi} f\left(z^{\prime}\right) u\left(z^{\prime}\right)^{*}\left[R^{2}-\left(a-z_{o}\right)\left(a-z_{o}\right)^{*}\right] u\left(z^{\prime}\right) d \theta^{\prime} \quad\left(z^{\prime}=\bar{z}\right) \\
& =\frac{1}{2 \pi} \int_{0}^{2 \pi} f(z) u(z)^{*}\left[R^{2}-\left(a-z_{o}\right)\left(a-z_{o}\right)^{*}\right] u(z) d \theta
\end{aligned}
$$

On a $\tilde{u}(\tilde{a}-\tilde{\mathbf{z}})=(\tilde{a}-\tilde{\mathbf{z}}) \tilde{u}=\tilde{1}$ dans l'algèbre $\mathcal{C}^{\infty}(\Omega, A / \alpha)$ et $\tilde{a}=\tilde{a}^{*} \operatorname{donc} \tilde{a}, \tilde{a}^{*}, \tilde{u}, \tilde{u}^{*}$ commutent entre eux dans l'algèbre $\mathcal{C}^{\infty}(\Omega, A / \alpha)$, donc $\tilde{u}^{*}\left[\tilde{R}^{2}-\left(\tilde{a}-\tilde{z}_{o}\right)\left(\tilde{a}-\tilde{z}_{o}\right)^{*}\right] \tilde{u}=$ $\tilde{u}\left[\tilde{R}^{2}-\left(\tilde{a}-\tilde{z}_{o}\right)\left(\tilde{a}-\tilde{z}_{o}\right)^{*}\right] \tilde{u}^{*}$ dans l'algèbre $\mathcal{C}^{\infty}(\Omega, A / \alpha)$. D'où $\tilde{b}^{*}=\tilde{b}$. 
Soient $A / \alpha$ et $B / \beta$ deux algèbres de Banach involutives quotients.

$L: A \longrightarrow B$ un morphisme d'algèbres involutives unitaires et continu tel que $L(\alpha) \subset \beta$. $L$ induit le morphisme $\tilde{L}: A / \alpha \longrightarrow B / \beta$ d'algèbres involutives unitaire qui à $\tilde{a}$ associe $\tilde{L}(\tilde{a})=\tilde{L}(a)$.

\section{Proposition 9 :}

Sous les hypothèses précédentes, soient $\tilde{a} \in A / \alpha$ et $U$ un ouvert de $\mathbb{C}$ tel que :

$s p_{\alpha} a \subset B\left(z_{o}, R\right) \subset \bar{B}\left(z_{o}, R\right) \subset U,\left(z_{o} \in U, R>0\right)$. Soit $\tilde{f}$ un élément de $h(U, A / \alpha)$.

On pose $: g=L o f: U \longrightarrow B$.

Alors $g$ est harmonique, $s p_{\beta} L(a) \subset s p_{\alpha} a$ et $\tilde{g}(\tilde{L}(\tilde{a}))=\tilde{L}(\tilde{f}(\tilde{a}))$.

\section{Preuve :}

$L$ est linéaire continue donc $\triangle(g)=\triangle(L o f)=L o \triangle(f)=0$ d'où $g$ est harmonique.

Soit $z \notin s p_{\alpha} a$ alors il existe $b \in A, x \in \alpha, y \in \alpha$ tels que : $(a-z) b+x=$ $b(a-z)+y=1$. Donc $(L(a)-z) L(b)+L(x)=L(b)(L(a)-z)+L(y)=1$, or $L(\alpha) \subset \beta$ donc $z \notin s p_{\beta} L(a)$. D'où $s p_{\beta} L(a) \subset s p_{\alpha} a$. On a $(\tilde{a}-\tilde{\mathbf{z}}) \tilde{u}=\tilde{u}(\tilde{a}-\tilde{\mathbf{z}})=\tilde{1}$ dans l'algèbre $\mathcal{C}^{\infty}\left(\mathbb{C} \backslash s p_{\alpha} a, A / \alpha\right)$.

On pose $: v=L o u$, le théorème du graphe fermé donne $L: \alpha \longrightarrow \beta$ continue.

$\operatorname{Donc}(\tilde{L}(\tilde{a})-\tilde{\mathbf{z}}) \tilde{v}=\tilde{v}(\tilde{L}(\tilde{a})-\tilde{\mathbf{z}})=\tilde{1}$ dans l'algèbre $\mathcal{C}^{\infty}\left(\mathbb{C} \backslash s p_{\alpha} a, A / \beta\right)$, parsuite on a $\tilde{g}(\tilde{L}(\tilde{a}))$ est la classe d'équivalence modulo $\beta$ de

$$
\begin{aligned}
& \frac{1}{2 \pi} \int_{0}^{2 \pi} \operatorname{Lof}(z) \operatorname{Lou}(z)\left[R^{2}-\left(L(a)-z_{o}\right)\left(L(a)-z_{o}\right)^{*}\right](\operatorname{Lou}(z))^{*} d \theta \\
& =L\left[\frac{1}{2 \pi} \int_{0}^{2 \pi} f(z) u(z)\left[R^{2}-\left(a-z_{o}\right)\left(a-z_{o}\right)^{*}\right] u(z)^{*} d \theta\right] \text {, car } L \text { est un morphisme }
\end{aligned}
$$
d'algèbres involutives continu unitaire. D'où $\tilde{g}(\tilde{L}(\tilde{a}))=\tilde{L}(\tilde{f}(\tilde{a}))$. 
Calcul fonctionnel harmonique dans une algebre de Banach involutive quotient 535

\section{Références}

[1] M.AKKAR H.ARROUB Applications du calcul fonctionnel harmonique dans une algèbre de Banach involutive quotient. A paraître dans Bulletin of The Belgian Mathematical Society Simon Stevin.

[2] A.ELKINANI Fonctions harmoniques opérant sur les algèbres de Banach involutives. Annales de l'Institut Fourier. Université Joseph Fourier. Grenoble. Tome 41_Fascicule 2 (1991), 493_509.

[3] J.W.M.FORD A square root lemma for * algebras.J. London. Math. Soc. 42 (1967), 521_522.

[4] L.WAELBROECK Les quotients banachiques . C. R. Acad. Sc. Paris. 286 1978), 37_39.

[5] L.WAELBROECK Quotient Banach spaces. Spectral. Theory. Banach center publications. Volume 8 PWN_Polish Scientific Publishers . Warsaw (1982), $553 \_562$

[6] L.WAELBROECK Holomorphic functional calculus and quotient Banach algebras . Studia Mathematica, T LXXV (1983) 273_286.

Université Bordeaux I

U.F.R. Mathématiques et Informatique

33405 Talence Cedex (France) 